\title{
Apport de l'analyste lors d'intoxications par les nouvelles drogues de synthèse
}

\section{Contribution of the analytical toxicologist in cases of poisoning with new synthetic drugs}

\section{Alain VERSTRAETE(1), Marc DEVEAUX ${ }^{(2)}$}

(1) Laboratoire de Biologie Clinique-Toxicologie, Hôpital Universitaire, De Pintelaan 185 B-9000 GENT - Belgique - Tél : + 3292403407 - Fax : + 3292404985 - mél : alain.verstraete@ rug.ac.be

(2) Institut de Médecine Légale, Place Théo Varlet - 59000 LILLE - France Tél : + 33 (0)320621223 - Fax : + 33 (0)320621229 - mél : mdeveaux@easynet.fr

(Reçu le 20 septembre 2000 ; accepté le 15 octobre 2000)

\section{$R E ́ S U M E ́$}

L'usage festif des nouvelles drogues de synthèse nécessite rarement le recours aux soins. Cependant la méconnaissance de la nature, de la dose et de la pureté des produits fait courir le risque d'un surdosage dramatique. Il est important de savoir reconnaître cliniquement une intoxication aiguë par les dérivés amphétaminiques regroupés sous le vocable d'ecstasy, par la kétamine ou encore par le gamma-hydroxybutyrate. L'aide de l'analyste est alors très précieuse pour confirmer la nature exacte de la ou des molécules en cause. Son apport est indispensable dans le cas de l'identification des isomères actifs ou lorsqu'il existe des métabolites communs non différenciés par l'immunoanalyse. Seule l'analyse systématique du sang et de l'urine par des méthodes chromatographiques avec identification spectrale permettra d'affirmer le diagnostic et indirectement d'avoir une meilleure connaissance des produits circulant sur le marché clandestin.

\section{MOTS-CLÉS}

Drogues de synthèse, usage festif, intoxication.

\section{SUMMARY}

Recreative use of new synthetic drugs rarely leads to acute health problems. However, the lack of knowledge of these products, their variable dose and purity can lead to severe overdose. It is important to be able to recognise clinically an acute poisoning with designer amphetamines (generally designated as ecstasy), ketamine and gamma-hydroxybutyrate $(G H B)$. The help of the analytical toxicologist is then needed to confirm the exact nature of the molecule $(s)$ involved. His contribution is essential in the case of identification of active isomers or when common metabolites exist, that are not differentiated with immunoassays. Only systematic toxicological analysis of blood and urine by chromatographic methods with spectrometric identification will allow for a better diagnosis and by this way to a better knowledge of the products that are available clandestinely.

\section{KEY-WORDS}

Designer drug, recreative use, poisoning. 


\section{Introduction}

Un ensemble de produits aux appellations mal contrôlées a fait son apparition depuis une dizaine d'années dans le milieu festif européen, notamment en Belgique et en France. C'est un nouveau paysage toxicologique qui s'est ainsi constitué peu à peu (1). Les raves parties sont les lieux de prédilection de cette polyconsommation, mais ils ne sont pas les seuls car les discothèques offrent aussi aux trafiquants un marché très important (2). Il est maintenant bien établi que les participants aux fêtes techno sous-estiment les risques d'intoxication aiguë qu'ils courent : la méconnaissance des produits utilisés peut conduire à une consommation répétitive et induire ainsi des états psycho-pathologiques aigus, tendant parfois vers la chronicité (3). Ce sont ces accidents aigus, somatiques ou psychiques, qui risquent de poser des problèmes de diagnostic aux urgentistes, encore moins au fait de ces nouvelles substances ou de ces nouveaux usages que leurs patients. L'identification du produit absorbé ne peut pas se faire de façon efficace sur le terrain et le patient et ses proches sont le plus souvent incapables de décrire précisément les symptômes : il importe donc que le laboratoire de toxicologie puisse identifier et au besoin doser dans les liquides biologiques le principe actif et/ou les métabolites. Le dialogue clinicien-analyste va alors permettre un diagnostic puis un traitement efficace. Nous proposons des exemples de démarches analytiques, en renvoyant au besoin le lecteur à des références détaillant précisément les méthodes analytiques. Seront étudiées les phényléthylamines stimulantes, entactogènes et hallucinogènes, puis la kétamine, et enfin le gamma-hydroxybutyrate (GHB) et ses analogues.

\section{Les symptômes des intoxi- cations par les nouvelles drogues de synthèse}

Les intoxications par les nouvelles drogues de synthèse vont se manifester par une psychostimulation accompagnée d'effets sympathomimétiques ou par une sédation et/ou des hallucinations $(4,5)$.

\section{Psychostimulation et effets sympathico- mimétiques}

Il se caractérise par les signes suivants : agitation, angoisse, paranoïa, panique, hallucinations, tachycardie, hypertension, hyperthermie, hypersudation, piloérection, mydriase, déshydratation, sensation de soif intense, sécheresse de la bouche, nausées, bruxisme, mouvements involontaires et hyperréflexie. Des convulsions, une hypotension et des troubles du rythme cardiaque peuvent être observés dans les cas graves. Ce syndrome sympathicomimétique peut être causé notamment par la cocaïne mais aussi par l'amphétamine, la méthamphétamine, la 3,4-méthylène dioxymùthylamphétamine (MDMA, ecstasy), la 3,4-méthylène dioxyéthylamphétamine (MDEA), la 3,4-méthylène dioxyamphétamine (MDA), la méthylbenzodioxazolylbutanamine (MBDB), la 2,5-diméthoxy-4-bromoamphétamine (DOB) et leurs analogues, ainsi que par certains décongestionnants nasaux comme la phénylpropanolamine, l'éphédrine et la pseudoéphédrine.

La classification pharmacologique de ces dérivés de la phényléthylamine se superpose pratiquement à leur classification chimique. Ce sont des substances permettant d'améliorer les performances. On distingue donc les amphétamines stimulantes (amphétamine et méthamphétamine), entactogènes (dérivés 3,4 méthylènedioxy : MDA, MDMA, MDEA, BDB et MBDB) et hallucinogènes (dérivés méthoxylés : $\mathrm{DOB}$, STP, TMA, PMA, 2C-B ; thioamphétamines : 2C-T-2 et 4MTA) (6). Tous les schémas métaboliques ne sont pas complètement élucidés, mais la MDA est un métabolite commun à de nombreuses molécules.

\section{Sédation}

Il comporte un myosis, une diminution de la motilité gastrique, une hypotension, de la bradycardie, de l'hypothermie, une hyporéflexie, une hypotonie, une somnolence, une dépression respiratoire, un œdème pulmonaire et peut se terminer par un coma. Une sédation profonde est observée lors d'une intoxication aiguë par l'alcool, les benzodiazépines, les barbituriques, les opioïdes et le GHB.

Le GHB est utilisé par voie IV comme anesthésique, à la posologie moyenne de $60 \mathrm{mg} / \mathrm{kg}$. Son utilisation détournée est argumentée sur des effets supposés ou réels : augmentation des taux d'hormone de croissance, euphorie, amnésie lacunaire (7). Des doses supérieures à $200 \mathrm{mg} / \mathrm{kg}$ par voie orale pourraient provoquer une détresse respiratoire. Les premiers effets euphorisants apparaissent en moins de 15 minutes et durent environ 30 minutes. Le retour à la normale s'effectue au bout de 2 à 4 heures. Il y a potentialisation par l'alcool éthylique.

Le 1,4-butanediol (BDO) et la gamma-butyrolactone (GBL) sont des précurseurs de la synthèse du GHB (in vitro et in vivo) et procurent les mêmes effets. On les considère cependant comme moins toxiques s'ils sont utilisés directement (8). La gamma-valérolactone (4- 
méthyl-GHB, GVL) est un analogue qui a été observé récemment. Le nom souvent utilisé de 'liquid ecstasy' pour désigner le GHB peut prêter à confusion et faire penser à la prise d'une amphétamine.

\section{Hallucinations}

Elles sont visuelles et auditives. On décrit aussi des phénomènes synesthésiques, c'est-à-dire l'enchaînement d'illusions d'un domaine sensoriel vers un autre. Les hallucinations peuvent être causées par le LSD, la psilocybine, la mescaline, les amphétamines hallucinogènes (MDMA, MDEA et MDA, DOB), les cannabinnoïdes, les tryptamines et des composés pipéridiniques comme la phencyclidine et la kétamine.

La kétamine (2-(o-chlorophényl)-2-(méthylamino)cyclohexanone) est un anesthésique à usage humain mais surtout vétérinaire, consommé en usage festif par voie IV mais aussi par voie orale et nasale à la dose de 200 à $500 \mu \mathrm{g}$. Ses propriétés pharmacologiques et pharmacocinétiques sont spécifiques, avec une grande rapidité d'action (5-10 min.), permettant un réveil rapide (l'effet dure 1 à 2 heures). Elle n'a pas d'effet convulsivant. Parmi ses huit métabolites connus, seules la norkétamine et la déhydronorkétamine sont actives, mais faiblement (9). En cas d'intoxication, on observe un tableau clinique caractéristique : immobilité, sédation, analgésie, dissociation corps-esprit. Il y a une augmentation du tonus musculaire, pouvant aller jusqu'à la catatonie (8) ainsi qu'une hyperthermie, un larmoiement et une hypersialorrhée. L'administration trop rapide peut être la cause d'une apnée brutale.

\section{Analyse}

Une analyse toxicologique est indiquée pour trouver la cause des syndromes décrits ci-dessus, mais aussi pour exclure une cause toxicologique à ces syndromes.

\section{Amphétamines}

Pour la recherche des amphétamines dans les urines, de nombreux kits d'immunoanalyse sont disponibles. Il faut interpréter avec prudence leurs résultats car de nombreux faux-positifs et faux-négatifs ont été décrits (10). Les décongestionnants nasaux et les anorexigènes peuvent causer des faux positifs. Certains composés amphétaminiques ne sont pas bien détectés par tous les kits, sauf à concentration élevée (11). Pour la confirmation et l'identification des amphétamines, la méthode la plus utilisée est la chromatographie en phase gazeuse couplée à la spectrométrie de masse (CG-SM) (12). Lors de la confirmation, l'analyste ne doit pas se limiter à rechercher les substances les plus connues. Certaines suggestions pour aider à l'identification d'un spectre inconnu peuvent être faites : par exemple, la 4méthylthioamphétamoine (4-MTA), apparue en 1998, est relativement facile à détecter car elle donne un résultat positif lors du dépistage des amphétamines par FPIA. A l'hôpital universitaire de Gand, cinq cas d'intoxication à la 4-MTA ont été observés. Dans l'un d'eux, un spectre inconnu lors de la confirmation des amphétamines à pu être identifié par comparaison avec celui de la 4-MTA proposé sur le site web du London Toxicology Group. Plus tard, un étalon a pu être obtenu pour confirmer cette première identification. Dans le cadre législatif, et c'est notamment le cas aux EtatsUnis pour la méthamphétamine, l'identification des isomères pourrait s'avérer nécessaire : une séparation chirale peut être effectuée par CG-SM ou CLHP (12) ou encore par électrophorèse capillaire (13).

\section{Kétamine}

Les techniques de dosage de la kétamine sont la CLHP, la CG-NPD et la CG-SM. La méthode CLHP permet de séparer les énantiomères. L'extraction se fait par le cyclohexane sur le plasma alcalinisé. La séparation stéréosélective est effectuée sans dérivation sur une colonne alpha1-glycoprotéine acide (14). En utilisant une colonne à polarité de phase inversée et après une ré-extraction en milieu acide ( $\mathrm{HCl} 1 \mathrm{M}$ ) pour éliminer les interférences, il est possible de doser la kétamine et la norkétamine (14). Si l'on travaille en CG-NPD, aucune dérivation n'est nécessaire. L'extraction liquideliquide se fait en une étape et la durée de l'analyse est de 5 minutes (15).

La méthode par CG-SM utilise une extraction liquideliquide par le mélange hexane:dichlorométhane (4:1 $\mathrm{vol} / \mathrm{vol})$. Les ions $238(\mathrm{M}+1$ de la kétamine) et 275 sont sélectionnés pour la quantification (16).

\section{GHB}

L'analyse du GHB dans les liquides biologiques n'est pas aisée. Aucun kit d'immunoanalyse n'est disponible sur le marché. Le GHB est volatil et se transforme en gamma-butyrolactone (GBL) en milieu acide (17). Un test colorimétrique a été proposé récemment (18). La chromatographie en phase liquide haute performance avec détection UV n'est pas très sensible et ne peut être utilisée que pour les substances saisies (19) ou les concentrations élevées, même si récemment, une limite de quantification de $10 \mu \mathrm{g} / \mathrm{mL}$ a été atteinte (20). La méthode la plus utilisée est la chromatographie en phase gazeuse avec détection par ionisation de flamme 
(17), capture d'électrons (21) ou spectrométrie de masse (22). Le plus souvent, le GHB est converti en GBL par un acide (22). Une extraction liquide-liquide (par exemple avec du dichlorométhane (23)) ou une micro-extraction en phase solide dans l'espace de tête peut être utilisée (24). Une autre alternative consiste à faire une extraction du GHB puis à procéder à son dosage par CG ou CG-SM après dérivation $(25,26)$. Récemment, une méthode CG-SM qui combine une extraction en phase solide et une extraction liquideliquide a été décrite (27).

Comme le GHB est présent dans l'organisme par production endogène, les seuils de positivité suivants sont utilisés : $2 \mu \mathrm{g} / \mathrm{mL}$ dans le sérum et $10 \mu \mathrm{g} / \mathrm{mL}$ dans les urines.

Dans certains cas, il sera plus facile d'identifier la substance si on dispose du produit qui a été ingéré (28).

\section{Conclusion}

Les amphétamines et la majorité de leurs dérivés sont assez faciles à détecter par immunoessai et à identifier par CG-SM. Ceci n'est pas toujours le cas, par exemple pour la $\mathrm{DOB}$, consommée à dose très faible et dont la limite de détection par FPIA est de $9000 \mathrm{ng} / \mathrm{mL}$ (29). En cas de symptômes suggestifs, il faut confirmer l'identification des amphétamines par une méthode sensible et spécifique, même si le dépistage est négatif. Souvent, une dérivation sera nécessaire pour améliorer la sensibilité. Les caractéristiques des spectres de masse des amphétamines dérivées ont été publiées (29). La kétamine est assez facile à détecter par les méthodes de screening chromatographiques comme la CG-SM ou la CLHP. Par contre le GHB et ses précurseurs ne sont pas détectables par les méthodes clas- siques, et devront être recherchés spécifiquement lorsque la clinique sera suggestive (coma profond avec un réveil rapide).

Dans les cas où l'analyse de routine n'aura pas trouvé d'explication aux symptômes observés chez le patient, une recherche plus approfondie sera nécessaire. Un screening large d'après les méthodes décrites par Maurer (30) ou Battu (31) peut être pratiqué. Dans ce cas, il peut également être utile de s'informer sur les nouvelles molécules qui ont été observées lors de saisies ou lors de l'identification sommaire des pilules d'ecstasy sur le terrain (testing). De bonnes sources d'informations sont :

- Le site du London Toxicology Group (www.londontox .org), qui contient des informations sur les nouvelles drogues apparues en Europe, utiles pour les cliniciens et pour les analystes (spectres de masse, détectabilité par immunoanalyse) ;

- www.ecstasy.org/testing/db/index.html : résultats du testing (test de Marquis et parfois identification plus poussée), avec description des effets perçus par les usagers ;

- www.checkyourdrugs.at/ : projet Check-It en Autriche, qui teste les pilules et publie les dernières informations sur les substances retrouvées ;

- Observatoire Européen des Drogues et Toxicomanie (EMCDDA, www.emcdda.org). On peut télécharger leurs publications, entre autres celles sur les risques associées à l'utilisation des nouvelles drogues de synthèse comme le MBDB et la 4-MTA.

Une bonne collaboration entre le clinicien et l'analyste est donc indispensable pour mener à bien cette recherche.

\section{Références}

1. Ingold R., Toussirt M. Ecsta, trip, coke et speed. Courr. Addictions. $1999 ; 1$ : 163-4.

2. Colombié T., lalam N., Schiray M. Drogue et techno, les trafiquants de rave. Paris : Stock, $2000: 125 \mathrm{pp}$.

3. Collectif. Usages de drogues de synthèse et réduction des risques dans le milieu festif techno. Courr. Addictions. $2000 ; 2$ : 33-9.

4. Ellenhorn M.J. Ellenhorn's medical toxicology. Baltimore : Williams and Wilkins, 1997 ; 3-46.

5. Vincent F. Hallucinogènes. in Toxicologie et pharmacologie médicolégales, Kintz P. (coord.), Paris :Elsevier Option Bio, 1998 : 555 - 93.

6. Deveaux M., Gosset D. Les psychotropes haute couture. Ann. Toxicol. Anal. $2000 ; 12$ : 90-6.
7. Ghysel M.H. Le GHB : l'acide gamma hydoxy butyrique. Revue de la littérature. Toxicorama. 1999 ; 11 : 1-11.

8. Walls H.C. GHB, ketamine and MDMA. in : Drugs, driving and traffic safety workshop. SOFT annual meeting, Puerto Rico, octobre 1999.

9. Fishbein L. Chromatography of environmental hazards. Vol.4 Drugs of abuse. Amsterdam : Elsevier, 1982 ; 473 9.

10. Verstraete A., Van Haute I. Spécificité des immunoessais pour la détection des amphétamines. Toxicorama 1997; 9 (1) : 65-71.

11. Verstraete A., Pezzati P., Van de Velde E.J. Comparison of six immunoassays for the detection of amphetamines and ecstasy. Communication, SOFT annual meeting, Milwaukee, octobre 2000. 
12. Kraemer T., Maurer H.H. Determination of amphetamine, methamphetamine and amphetamine-derived designer drugs or medicaments in blood and urine. $\mathrm{J}$. Chromatogr. B 1998; 713 : 163-87.

13. Labat L., Deveaux M., Dubost J.-P. Applications de l'électrophorèse capillaire en toxicologie clinique et médico-légale. Ann. Toxicol. Anal. 2000; 12 (3) : 179-95.

14. Geisslinger G., Menzel-Soglowek S., Kamp H.D., Brune $\mathrm{K}$. Stereoselective high-performance liquid chromatographic determination of the enantiomers of ketamine and norketamine in plasma. J Chromatogr 1991; 568 : 165-76.

15. Kintz P., Mangin P., Lugnier A.A., Chaumont A.J. Dosage de la kétamine dans les liquides biologiques. Cah Anesthesiol 1990; 38 : 173-4.

16. Feng N., Vollenwider F.X., Minder E.I., Rentsch K., Grampp T., Vonderschmitt D.J. Development of a gas chromatography-mass spectrometry method for determination of ketamine in plasma and its application to human samples. Ther Drug Monit 1995; 17 : 95-100.

17. Letteri J., Fung H. Evaluation and development of gas chromatographic procedures for the determination of gamma-hydroxybutyric acid and gamma-butyrolactone in plasma. Biochem. Med. 1978; $20: 70-80$.

18. Badcock N.R., Zotti R. Rapid screening test for gammahydroxybutyric acid (GHB, Fantasy) in urine. Ther. Drug Monit. $1999 ; 21: 376$

19. Mesmer M.Z., Satzger R.D. Determination of gammahydroxybutyrate (GHB) and gamma-butyrolactone (GBL) by HPLC/UV-VIS spectrophotometry and HPLC/thermospray mass spectrometry. J. Forensic Sci. $1998 ; 43: 1259-60$.

20. De Vriendt C.A., Van Sassenbrouck D.V., Rosseel M.T., Van De Velde E., Verstraete A.G., Vander Heyden Y., Belpaire F.M. Development and validation of an HPLC method for the determination of gamma-hydroxybutyric acid (GHB) in rat plasma. J. Chrom B. (In press)

21. Doherty J., Snead O., Roth R. A sensitive method for quantitation of gamma-hydroxybutyric acid and gammabutyrolactone in brain by electron capture gas chromatography. Anal. Biochem. 1975; 69 : 268-77.

22. Ferrara S.D., Tedeschi L., Frison G., Castagna F., Gallimberti L., Giorgetti R., Gessa G.L., Palatini P. Therapeutic gamma-hydroxybutyric acid [4-hydroxybutyric acid] monitoring in plasma and urine by gas chromatography-mass spectrometry. J. Pharm. Biomed. Anal. 1993; $11: 483-7$.
23. Thomas B., Schöntube E. Gaschromatographische Bestimmung von Gamma-Hydroxybüttersäure in human Plasma. Pharmazie. 1993; 48 : 623-4.

24. Frison G. Tedeschi L. Maietti S, Ferrara S. D. Determination of gamma-hydroxybutyrate (GHB) in plasma and urine by headspace solid-phase microextraction (SPME) and gas chromatography-positive ion chemical ionization-mass spectrometry. in : Spiehler V. Proceedings of the SOFT/TIAFT International Meeting. 1999 : Newport Beach, 394-404.

25. Verstraete A.G., Van de Velde E., De Paepe P. Determination of gamma-hydroxtbutyrate in serum or plasma with GC/MS. In: Lech T. Proceedings of the 37th TIAFT Meeting. Problems in Forensic Sciences XLII. Cracow, 2000 : 195-201.

26. Couper F.J., Logan B.K. Determination of gammahydroxybutyrate (GHB) in biological specimens by gas chromatography-mass spectrometry. J. Anal. Toxicol. 2000; $24: 1-7$.

27. McCusker R.R., Paget W.H., Chronister C.W., Goldberger B.A., ElSohly M.A. Analysis of gammahydroxybutyrate (GHB) in urine by gas chromatography-mass spectrometry. J. Anal. Toxicol. $1999 ; 23$ : 301-5.

28. Louagie H.K., Verstraete A.G., De Soete C.J., Baetens D.G., Calle P.A. A sudden awakening from a near coma after combined intake of gamma-hydroxybutyric acid (GHB) and ethanol. J. Toxicol. Clin. Toxicol. 1997; 35 : 591-4.

29. Ghysel M.H. Amphétamines et dérivés. in : Toxicologie et pharmacologie médicolégales, Kintz P. (coord.), Paris : Elsevier Option Bio, 1998 : 466-541.

30. Maurer H.H. Systematic toxicological analysis of drugs and their metabolites by gas chromatography - mass spectrometry. J Chromatrogr. B. 1992; 580 : 3-41.

31. Battu C., Marquet P., Fauconnet A.-L., Lotfi H., Lachâtre G. Screening de 21 amphétamines dans l'urine par micro-extraction en phase solide (SPME) et chromatographie en phase gazeuse -spéctrométrie de masse (CGSM). Toxicorama. $1997 ; 9$ (2) : 73-81. 